http://dx.doi.org/10.35630/2199-885X/2020/10/2.15

\title{
POST-TRAUMATIC DAMAGE OF BRACHIAL PLEXUS: CLINICAL CASE STUDY
}

\section{Oleg Zayko ${ }^{1 凶}$ (D), Levon Turadzhyan ${ }^{1}$, Vadim Astashov ${ }^{1}$, Anna Sindireva ${ }^{2}$ (D), Svetlana Smolina ${ }^{1}$, Karina Basnakyan ${ }^{1}$}

\author{
${ }^{1}$ RUDN University, Moscow, \\ ${ }^{2}$ Tyumen State University, Tyumen, Russia
}

oleg.zayko@bk.ru
ABSTRACT - The article presents a clinical case of a posttraumatic injury of branchial plexus. As a result of head-on car collision the driver suffered an injury of left brachial plexus and its branches, multiple rib fractures, a broken left collarbone. Formation of posttraumatic edema ultimately led to interruption of venous and lymphatic drainage from the left axillary basin. A posttraumatic keloid and unremoved bone pieces aggravated the manifestations of edema, which persisted 6 months. This strongly challenged the rehabilitation aimed to restore nerve conduction.

KEY W ORDS - brachial plexus injury, lymphedema, tissue hypoxia, lipid peroxidation.

\section{INTRODUCTION}

A brachial plexus injury is one of the most common peripheral nerve injuries. Specific features of preganglionic traumatic injury allow prediction of low likelihood of spontaneous recovery, and the need for surgical intervention tends to be high [1]. Such injuries usually result in disordered motor function, compromised sensitive impulse conductivity, cause severe disability and dramatic decrease of quality of life $[3,4]$. Progressing hypoxia also hinders the normal recovery process. Post-traumatic pain shock, blood supply, lymph efflux and venous drainage disturbance contribute to worsening of existing tissue and circulatory hypoxia with subsequent oxidative stress development, accompanied by accumulation of lipid peroxidation products and damage of cellular membrane structures [5].

\section{Clinical Case History}

Patient, female, 48 years old, with the history of road traffic accident in September 2013 resulting in the severe brain contusion (post-traumatic encephalopathy with diffuse atrophy in the parietal lobe), multiple fractures of 1-4 ribs, fracture of the clavicle, compression
Received 15 May 2020;

Received in revised form 30 May 2020; Accepted 4 June 2020

spinal fracture with D3, D5 disk injury, brachial plexus rupture (monoplegia of left arm with complete loss of any sensitivity), detachment of nerve roots $\mathrm{C} 5-6$, C6-7, C7-Th1, Th1-2 on the left and C5-6, C6-7 on the right, subscapularis tendon injury and traumatic injury of musculus deltoideus fibers. The third upper phantom limb caused severe pain syndrome.

In April 2014, the patient underwent revision surgery and neurolysis of primary and secondary stems, including distal branches of the right brachial plexus. Use of intercostal nerves for restoration of nerve conductivity in the injured extremity was not feasible due to the injury-related intercostal nerve structural changes. During post-surgery period, the patient suffered from persistent edema of injured site that contributed to the local negative impact on the peripheral nerves. Treatment administered for edema was inefficient, which further adversely affected restoration of the nerve impulse conductivity. During the early post-surgical period, the patient was administered with treatment including pharmacological and physical therapies at the present day, due to total loss of motor function and sensitivity of the left upper extremity and progressing lateral curvature of vertebral spine caused by additional load on this area, it is recommended to amputate non-functional extremity.

\section{WORK-UP RESULTS}

MRI Results over Time

The first MRI scan made in 1 month after injury.

The second MRI scan made in 2 months after injury.

The third MRI scan made in 6 months after injury.

Blood Tests Results over Time

Interpretation of results if blood tests made immediately on the day of injury revealed no changes in the red blood cell count, hemoglobin $(\mathrm{Hb})$ concentration, and blood urea nitrogen concentration beyond normal range. At the same time, the creatinine concentration exceeded the upper normal limit by $7 \%$, which was explained by the tissue damage as a result of traffic accident. ESR also increased and exceeded the upper normal limit by $36 \%$. 


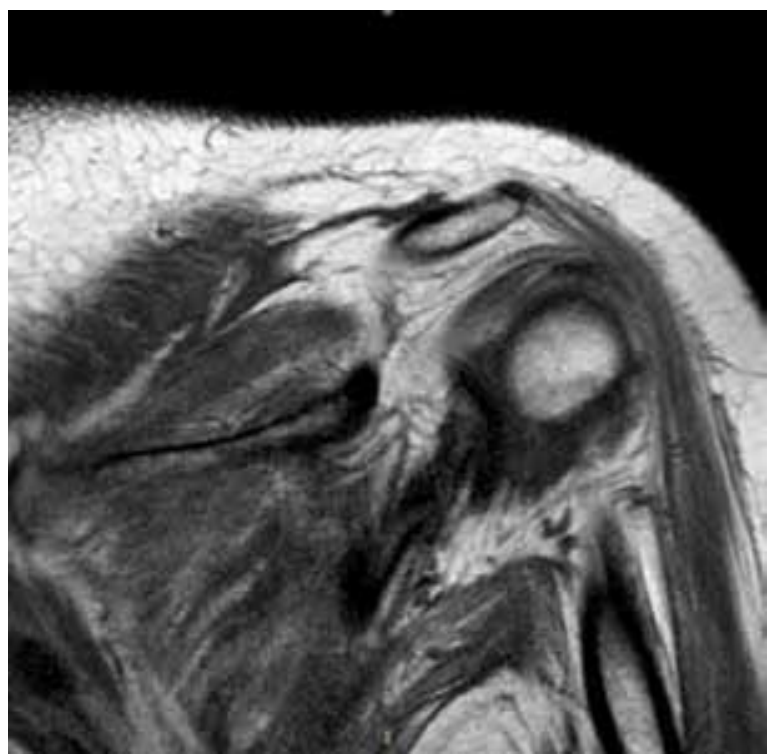

Fig. 1. The injured site exhibits reduced blood supply along the major branches of the left subclavian artery (in the interscalene space) and the axillary artery. The scan shows the area of edema with compression of $(5-T h 2$ roots. The sings of subclavian lymph vessels of $2-3$ orders, the signs of bone fragments and colloid scars are also observed. In addition, the features of left lung upper lobe compression (S1-2, S3) are demonstrated

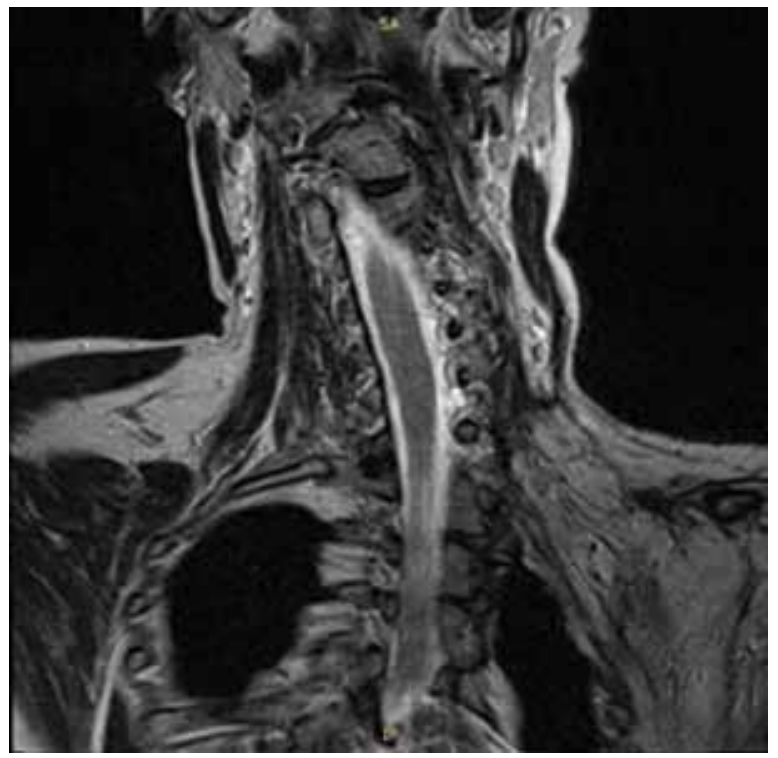

Fig. 2. The scan shows growing edema resulting from pressure on major blood vessels and their branches. The scar at the inured site exhibits no changes over time

Blood tests made in 2 months after the accident showed reduction of the white blood cell count to the normal level and ESR reduction to the level slightly

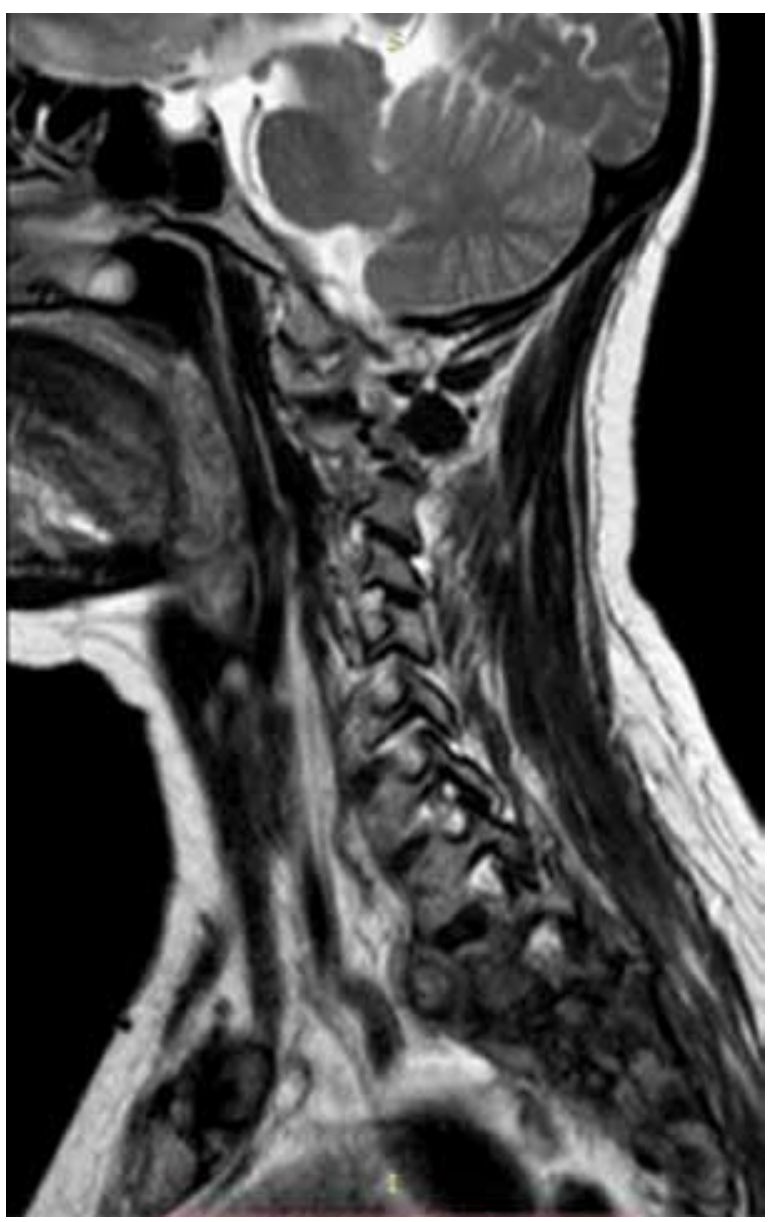

Fig. 3. The scan shows persistent edema and lymphatic ducts obstruction, compression of regional lymph nodes and vessels at the injured site, which contributes to the development of hypoxia and severe hemoglobinopenia

above the normal range. $\mathrm{Hb}$ concentration and red blood cell count decreased by $5 \%$ as compared to the blood test results as of the accident date. The blood urea nitrogen showed significant increase (by 38\%), which might result from activation of anaerobic glycolysis processes due to elevated glycogen depot consumption and inefficient aerobic glycolysis.

In 6 months after the injury, red blood cell count and ESR were within the normal range. The blood tests results revealed leukocytopenia (decrease by 6\% beyond the lower normal level) probably caused by administration of high doses of antibiotics. Creatinine and urea concentrations remained high and exceeded upper normal level by $25 \%$ and $13 \%$ respectively, while $\mathrm{Hb}$ concentration showed no appreciable change as compared to the blood tests results as of the second month after injury. These results were most probably due to intensification of tissue hypoxia as a result of 
failure to eliminate the reasons for its development: persistence of the coarse structural changes at the injured site, bone fragments, formation of colloid scars, post-traumatic anatomic asymmetry, which is confirmed by MRI data.

The persistent tissue hypoxia developed after the injury resulted in depletion of antioxidant pool, which led to enhancement of lipid peroxidation with accumulation of above reaction products at the injured site and subsequent cellular membrane destruction - in this particular case, trophic damage of the peripheral nerve tissue, preconditioned by failure to achieve nerve conductivity restoration (negative functional dynamics, decreased amplitude of injured extremity, worsening of pain syndrome and development of third limb phantom).

Taking into account the fact that the timeframes for nerve tissue restoration are usually within 3 months, in the first days after the injury it was imperative to direct the best possible efforts to efficiently combat the post-traumatic tissue edema, restore lymph efflux and adequate blood supply of this area and prevent formation of lipid peroxides.

\section{CONCLUSIONS}

It is important to note that the first medical aid for patients with brachial plexus injury should be directed to restore (preserve) anatomical structures at the damages site. It is necessary to start immediate anti-edema treatment in order to avoid hypoxia. During a surgery, it is important to prevent development of post-traumatic scars leading to blood supply, venous drainage and lymph drainage disruption [6,7]. In the early post-surgery period, it is crucial to achieve functional restoration of the injured site, which also contributes to reduction of hypoxia in the damaged area. It should be noted that this type of injuries mandates further more detailed research by morphophysiologists in order to determine the pattern of nerve structures damage at the cellular level and ensure possibility for complete restoration.

\section{REFERENCES}

1. Sharma MS. Restoring movements at the shoulder joint in pan-brachial plexus injuries: [Electronic resource]: Focusing on the complex kinesiology. / Sharma MS. - Electronic resource. - Department of Neurosurgery, Mayo Clinic Health System, Mankato, Minnesota, USA, 2019. doi: 10.4103/00283886.250711

2. Huan KWSJ, Tan JSW, Tan SH, Teoh LC, Yong FC. Restoration of shoulder abduction in brachial plexus avulsion injuries with double neurotization from the spinal accessory nerve [Electronic resource]: a report of 13 cases. - / Electronic resource. - Singapore, 2017. doi: 10.1177/1753193416680725.
3. LeECHAVENgVongs S1, MalungPaishorpe K2, Uerpairojkit C2, Ng CY3, Witoonchart K2. Nerve Transfers to Restore Shoulder Function. [Electronic resource] . - / Leechavengvongs S1, Malungpaishorpe K2, Uerpairojkit C2, Ng CY3, Witoonchart K2. - Electronic resource. - 1Department of Medical Services, Institute of Orthopaedics, Lerdsin General Hospital, 190 Silom Road, Bangrak, Bangkok 10500, Thailand. Electronic address: somsakortho@gmail. com. 2Department of Medical Services, Institute of Orthopaedics, Lerdsin General Hospital, 190 Silom Road, Bangrak, Bangkok 10500, Thailand. 3Upper Limb Unit, Wrightington Hospital, Hall Lane, Appley Bridge, Wigan WN6 9EP, UK, 2016. DOI: 10.1016/j. hcl.2015.12.004

4. Dor K. Distal Nerve Transfer [Electronic resource]: Perspective of Reconstructive Microsurger. - / Doi K. - Electronic resource. - Department of Orthopaedic Surgery, Ogori Daiichi General Hospital, Ogori, Yamaguchi, Japan, 2018. DOI: 10.1055/s-00381639369 